U.S. Department of the Interior

U.S. Geological Survey

\section{Ground-Water Quality near a Septage-Treatment Facility, Orleans, Massachusetts, 1997-98}

By LESLIE A. DESIMONE and KIRK P. SMITH

Open-File Report 00-285

Prepared in cooperation with the

MASSACHUSETTS DEPARTMENT OF ENVIRONMENTAL PROTECTION, DIVISION OF WATERSHED MANAGEMENT

Northborough, Massachusetts

2000 


\section{U.S. DEPARTMENT OF THE INTERIOR \\ BRUCE BABBITT, Secretary}

U.S. GEOLOGICAL SURVEY

Charles G. Groat, Director

The use of trade or product names in this report is for identification purposes only and does not constitute endorsement by the U.S. Geological Survey.

For additional information write to:

Chief, Massachusetts-Rhode Island District

U.S. Geological Survey

Water Resources Division

10 Bearfoot Road

Northborough, MA 01532

or through our web site at http://ma.water.usgs.gov
Copies of this report can be purchased from:

U.S. Geological Survey

Branch of Information Services

Box 25286

Denver, CO 80225-0286 


\section{CONTENTS}

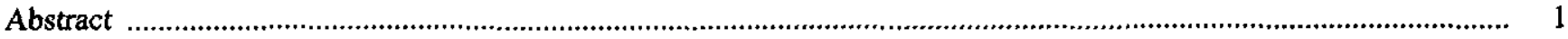

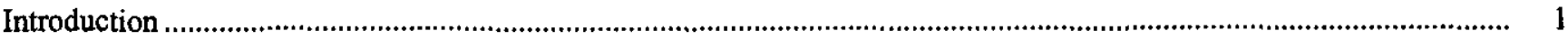

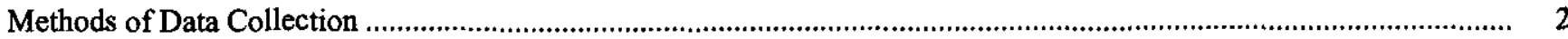

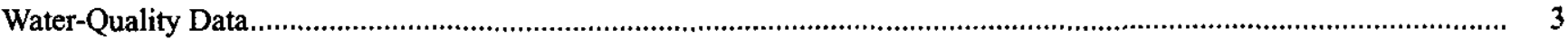

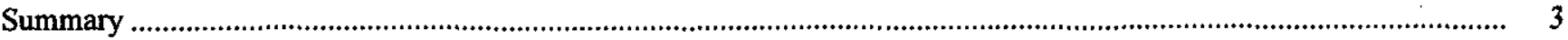

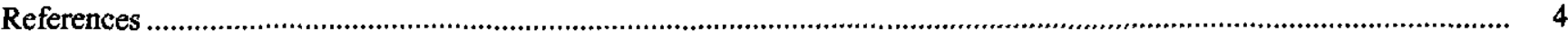

\section{FIGURES}

1. Map showing the location of well-cluster sites used for water-quality sampling, Tri-Town Septage

Treatment Facility, Orleans, Massachusetts.

\section{TABLES}

1. Location coordinates and land-surface and screen altitudes of sampled wells, Tri-Town Septage Treatment

Facility, Orleans, Massachusetts

2. Physical properties and concentrations of major inorganic constituents, nutrients, and dissolved organic

carbon in ground water near the Tri-Town Septage Treatment facility

3. Concentrations of trace metals in ground water near the Tri-Town Septage Treatment facility.

4. Concentrations of volatile organic compounds in ground water near the Tri-Town Septage Treatment facility

\section{CONVERSION FACTORS, VERTICAL DATUM, AND WATER-QUALITY INFORMATION}

\section{CONVERSION FACTORS}

\begin{tabular}{rll}
\hline Multiply & \multicolumn{1}{c}{ By } & To obtaln \\
\hline inch (in.) & 2.54 & centimeter \\
foot (ft) & 0.3048 & meter \\
gallon (gal) & 3.785 & liter \\
gallon per day (gal/d) & 0.003785 & cubic meter per day \\
mile (mi) & 1.609 & kilometer \\
Temperature is given in degrees Celsius $\left({ }^{\circ} \mathrm{C}\right)$, which can be converted to degres \\
& Fahrenheit $\left({ }^{\circ} \mathrm{F}\right)$ by the following equation: \\
& ${ }^{\circ} \mathrm{F}=\left({ }^{\circ} \mathrm{C}+32\right) \times 1.8$ & \\
\hline
\end{tabular}

\section{VERTICAL DATUM}

Sea level: In this report, "sea level" refers to the National Geodetic Vertical Datum of 1929 (NGVD of 1929) - a geodetic datum derived from a general adjustment of the first-order level nets of the United States and Canada, formerly called Sea Level Datum of 1929.

\section{WATER-QUALITY INFORMATION}

Concentrations of chemical constituents are give in milligrams per liter $(\mathrm{mg} / \mathrm{L})$ or micrograms per liter. Milligrams per liter and micrograms per liter are units expressing the concentration of a chemical constituent in solution as weight (milligrams or micrograms) of solute per unit volume (liter) of water. For concentrations less than $7,000 \mathrm{mg} / \mathrm{L}$, milligrams per liter is equivalent to "parts per million" and micrograms per liter is equivalent to "parts per billion". 


\title{
Ground-Water Quality near a Septage-Treatment Facility, Orleans, Massachusetts, 1997-98
}

\author{
By Leslie A. DeSimone and Kirk P. Smith
}

\section{Abstract}

Water-quality data were collected from 27 ground-water wells near a septage-treatment facility in Orleans, Cape Cod, Massachusetts, from November 1997 through March 1998. The data were collected to characterize a plume of nitrogen-rich ground water that has resulted from infiltration of treated septage effluent. The waterquality data include physical properties and concentrations of major inorganic constituents, nutrients, and dissolved organic carbon in ground water. Concentrations of trace metals and volatile organic compounds also were measured in ground water from three wells. Dissolved nitrate was the primary form of nitrogen in ground water near the treatment facility. Nitrate concentrations ranged from less than 0.1 milligram per liter as nitrogen in wells representative of background conditions to 74 milligrams per liter as nitrogen in a well screened at the water table beneath the effluent infiltration beds. Smaller amounts of dissolved ammonia and organic nitrogen also were present. Ground water with high nitrogen concentrations also had a high specific conductance, low dissolved oxygen, and high concentrations of major ions. Concentrations of trace metals and volatile organic compounds in water from the sampled wells were all below drinking water standards or below analytical detection limits.

\section{INTRODUCTION}

Infiltration of effluent from the Tri-Town Septage Treatment Facility in Orleans, Cape Cod, Massachusetts, has resulted in a plume of nitrogen-rich ground water in the stratified-drift aquifer (DeSimone and others, 1996). Effluent has discharged at about $20,000 \mathrm{gal} / \mathrm{d}$ since 1990 . The disposal area is about $1 \mathrm{mi}$ inland from Cape Cod Bay and about $700 \mathrm{ft}$ from coastal Namskaket Marsh, an Area of Critical Environmental Concern as designated by the Commonwealth of Massachusetts (fig. 1, at back of report). Because nitrogen can contribute to eutrophication of coastal waters, the potential discharge of the nitrogen-rich ground-water plume to these coastal water resources has been a concern to State and local water-resource managers, local conservation groups, and the general public.

The U.S. Geological Survey (USGS), in cooperation with the Massachusetts Department of Environmental Protection, has monitored the groundwater plume that has resulted from effluent discharge at the treatment facility since its inception. Monitoring was done through the installation of observation wells, water-quality sampling, and geophysical methods. Nitrogen geochemistry in the plume also was studied (DeSimone and Howes, 1996, 1998), and ground-water transport pathways, ecological conditions, and baseline water quality in Namskaket Marsh were investigated by the USGS in cooperation with the Woods Hole Oceanographic Institute and Cape Cod Commission (Howes and others, 1996; Weiskel and others, 1996, 
1997; DeSimone and others, 1998a, 1998b). Ground water was sampled frequently from 1990 through 1992 (DeSimone and Howes, 1995; DeSimone and others, 1996). Since 1992, movement of the ground-water plume through the aquifer has been monitored primarily with borehole electromagnetic-induction logging, which detects the zones of elevated electrical conductivity that are associated with the effluentcontaminated ground water (DeSimone and Barlow, 1994). Electromagnetic-induction logging indicates that by March 1997 the leading edge of the septageeffluent plume had advanced about $500 \mathrm{ft}$ west and 800 $\mathrm{ft}$ northwest from the infiltration beds toward potential coastal discharge areas (Weiskel and others, 1997). More recent logging suggests that the leading edge of the plume appears to have moved to a position that is horizontally within $50 \mathrm{ft}$ of the upland boundary of Namskaket Marsh (site 185, fig. 1) and is 5 to $10 \mathrm{ft}$ below marsh sediments (J.M. Norris, U.S. Geological Survey, written commun., 1998).

The water-quality data presented in this report were collected to provide an update on concentrations of nitrogen species and other chemical constituents of the plume and to verify inferences about the spatial extent of the plume from borehole-induction logs. Since 1992, when ground water in the plume was last sampled, the plume has increased in size, and the chemical characteristics of the effluent have changed due to changes in the septage-treatment procedures (James Burgess, Tri-Town Septage Treatment Facility, written commun., 1996-98). Thus, more recent information was needed to accurately describe waterquality conditions in the aquifer. Water-quality data were collected from 27 wells at 21 well-cluster sites from November 1997 to March 1998 (tables 1 and 2, at back of report and fig. 1). The sampled wells were located within and at the margins of the ground-water plume and are part of a larger observation-well network (DeSimone and Howes, 1995).

\section{METHODS OF DATA COLLECTION}

Water samples were collected from 2-inchdiameter, polyvinylchloride observation wells. Screened intervals of wells ranged from 44.5 to $129.9 \mathrm{ft}$ below land surface, or from $12.7 \mathrm{ft}$ above to $74.6 \mathrm{ft}$ below sea level. Samples for analysis of nutrients, major inorganic constituents, and dissolved organic carbon from most wells were collected with a stainless steel Keck Model SP-84 submersible pump fitted with Teflon tubing and an isolation packer. One well that was screened in low-permeability material, OSW 160, was sampled with a teflon bailer. Samples for analysis of trace metals and volatile organic compounds (VOCs) were collected with a Grundfos Model Redi-Flo2 submersible pump fitted with Teflon tubing. Samples for analysis of trace metals were collected and processed by two field-team members using procedures to minimize contamination as described by Koterba and others (1995). All samples were collected after at least three well-casing volumes were withdrawn.

Field properties- $\mathrm{pH}$, temperature, specific conductance, and dissolved oxygen-were monitored for stabilization during purging of each well. Field properties were measured with electrodes in a flow-through chamber with a Hydrolab model H-20 meter. For water samples with dissolved-oxygen concentrations less than $1 \mathrm{mg} / \mathrm{L}$, dissolved oxygen also was measured by means of a quantitative colorimetric method that uses self-filling reagent ampoules (Chemetrics Model R-7501).

The sampling pump and equipment were decontaminated with laboratory-grade deionized water after sampling at each well to prevent carry-over from well to well. The pumps and discharge lines were decontaminated by rinsing the pump and line exteriors and by passing 5 gal of deionized water through them. The sampling pump and equipment used to collect trace metal and VOC samples also were washed with 5 gal of 0.1 -percent Liquinox detergent solution followed by 20 gal of tap water before the final rinse 
with 5 gal of deionized water. Equipment for tracemetal sampling was stored and transported to the field site in polypropylene bags.

Samples were filtered and preserved, where needed, immediately after collection. Samples for analysis of nutrients and dissolved inorganic constituents were filtered through 0.45 -micron capsule filters (Gelman Model 12175) inline during pumping. Samples for analysis of dissolved organic carbon were filtered through 0.45 -micron silver filters with a stainless-steel, nitrogen-pressure filtration apparatus. Samples for analysis of cations and trace metals were preserved with nitric acid to $\mathrm{pH}$ less than 2 . Unfiltered samples for analysis of VOCs were collected into amber septum vials and preserved with hydrochloric acid. All sample bottles were sent on ice to the USGS National Water Quality Laboratory in Denver, Colorado, for analysis (Wershaw and others, 1987; Fishman and Friedman, 1989)

Samples also were collected for quality assurance and control. A rinse-water blank was prepared with the deionized water for analysis of nutrients and major inorganic constituents in November 1997. A field equipment blank also was collected in November 1997 for analysis of nutrients and major inorganic constituents by passing the deionized water through the sampling and processing equipment. A field equipment blank was collected in March 1998 using inorganic-free water for analysis of trace metals and organic-free water for analysis of dissolved organic carbon and VOCs. Finally, replicate samples were prepared from well OSW 168 for analysis of nutrients and major inorganic constituents and from well OSW 183 for analysis of trace metals.

\section{WATER-QUALITY DATA}

Physical properties and concentrations of major inorganic constituents, nutrients, and dissolved organic carbon are presented in table 2 . Nitrate was the primary form of nitrogen in ground water from the sampled wells. Concentrations of nitrate ranged from less than or equal to $0.1 \mathrm{mg} / \mathrm{L}$ as $\mathrm{N}$, at wells at the margins of the plume (OSW 111 and OSW 168, table 2), which is representative of background conditions (DeSimone and others, 1996), to $74 \mathrm{mg} / \mathrm{L}$ as $\mathrm{N}$ at a well screened near the water table beneath the infiltration beds (OSW 160, table 2). Smaller amounts of ammonia and organic nitrogen also were measured. Wells with high concentrations of dissolved nitrogen species also had high specific conductance, low dissolved oxygen, and high concentrations of major ions, including calcium, magnesium, sodium, and chloride (table 2).

Water samples from three wells were analyzed for concentrations of trace metals and VOCs (tables 3 and 4, at back of report). Aluminum, barium, chromium, cobalt, nickel, and zinc were detected at all three wells, but at concentrations that were less than drinking-water standards (U.S. Environmental Protection Agency, 1994). Of the suite of VOCs analyzed, three VOCs-chloroethane, chloromethane, and dichlorodifluoromethane-were detected, at concentrations below drinking-water standards. However, chloroethane and chloromethane also were detected in the equipment blank and may have been introduced during sampling collection.

\section{SUMMARY}

The data presented in this report represent the most recent detailed sampling of ground-water quality in the septage-effluent plume at the Tri-Town facility. These data should be useful to water-resource managers as the discharge from the facility and its potential effects on downgradient receptors continue to be regulated and monitored. The data also could be used to verify and further investigate the nitrogen transformations and other geochemical changes in the ground-water plume that were documented in earlier investigations at the Tri-Town site. 


\section{REFERENCES}

DeSimone, L.A., and Barlow, P.M., 1994, Borehole induction logging for delineation of a septage-effluent contaminant plume in glacial outwash, Cape Cod, Massachusetts, in Hallenberg, J.K., ed., Proceedings of the Fifth International Symposium on Geophysics for Mineral, Geotechnical, and Groundwater and Environmental Applications, Tulsa, Oklahoma, October 24-28, 1993, p. XI-XII.

DeSimone, L.A., Barlow, P.M., and Howes, B.L., 1996, A nitrogen-rich septage-effluent plume in a glacial aquifer, Cape Cod, Massachusetts, February 1990 through December 1992: U.S. Geological Survey Water-Supply Paper 2456, 89 p.

DeSimone, L.A., and Howes, B.L., 1995, Hydrogeologic, water-quality, and biogeochemical data collected at a septage-treatment facility, Orleans, Cape Cod, Massachusetts, October 1988 through December 1992: U.S. Geological Survey Open-File Report 95-429, 74 p. 1996, Denitrification and nitrogen transport in a coastal aquifer receiving wastewater discharge: Environmental Science and Technology, v. 30, p. 11521162.

1998, Nitrogen transport and transformations in a shallow aquifer receiving wastewater discharge: a mass balance approach: Water Resources Research, v. 34, no. 2, p. 271-285.

DeSimone, L.A., Howes, B.L., Goehringer, D.G., and Weiskel, P.K., 1998a, Wetland plants and algae in a coastal marsh, Orleans, Cape Cod, Massachusetts: U.S. Geological Survey Water-Resources Investigations Report 93-4011, 33 p.

DeSimone, L.A., Weiskel, P.K., Howes, B.L., and Smith, K.P., 1998b, Water-quality data collected in a coastal marsh, Orleans, Cape Cod, Massachusetts, March 1993 through January 1998: U.S. Geological Survey OpenFile Report 98-184, 38 p.
Fishman, M.J., and Friedman, L.C., eds., 1989, Methods for determination of inorganic substances in water and fluvial sediments: U.S. Geological Survey Techniques of Water-Resources Investigations, chap. Al, book 5 , $545 \mathrm{p}$.

Howes, B.L., Weiskel, P.K., Goehringer, D.D., and Teal, J.M., 1996, Interception of freshwater and transport of nitrogen from uplands to coastal waters: the role of salt marshes, in Nordstrom, K.F., and Roman, C.T., eds., Estuarine Shores: Evolution, Environments and Human Alterations: London, John Wiley and Sons, p. 287-310.

Koberba, M.T., Wilde, F.D., and Lapham, W.W., 1995, Ground-water data-collection protocols and procedures for the National Water-Quality Assessment Program: collection and documentation of water-quality samples and related data: U.S. Geological Survey Open-File Report 95-399, 113 p.

U.S. Environmental Protection Agency, 1994, National primary drinking water standards: Washington, D.C., Office of Water, EPA810-F-94-001A, 4 p.

Weiskel, P.W., DeSimone, L.A., and Howes, B.L., 1996, A nitrogen-rich septage-effluent plume in a coastal aquifer, marsh, and creek system, Orleans, Massachusetts: project summary, 1988-95: U.S. Geological Survey Open-File Report 96-111, 20 p.

1997, The Namskaket Marsh project: nitrogen transport and ecosystem characterization in a Cape Cod aquifer and salt marsh: Environment Cape Cod, v. 1, no. 2, p. 10-27.

Wershaw, R.L., Fishman, M.J., Grabbe, R.R., and Lowe, L.E., eds., 1987, Methods for determination of organic substances in water and fluvial sediments: U.S. Geological Survey Techniques of Water-Resources Investigations, chap. A3, book 5, $80 \mathrm{p}$. 
FIGURE AND TABLES 


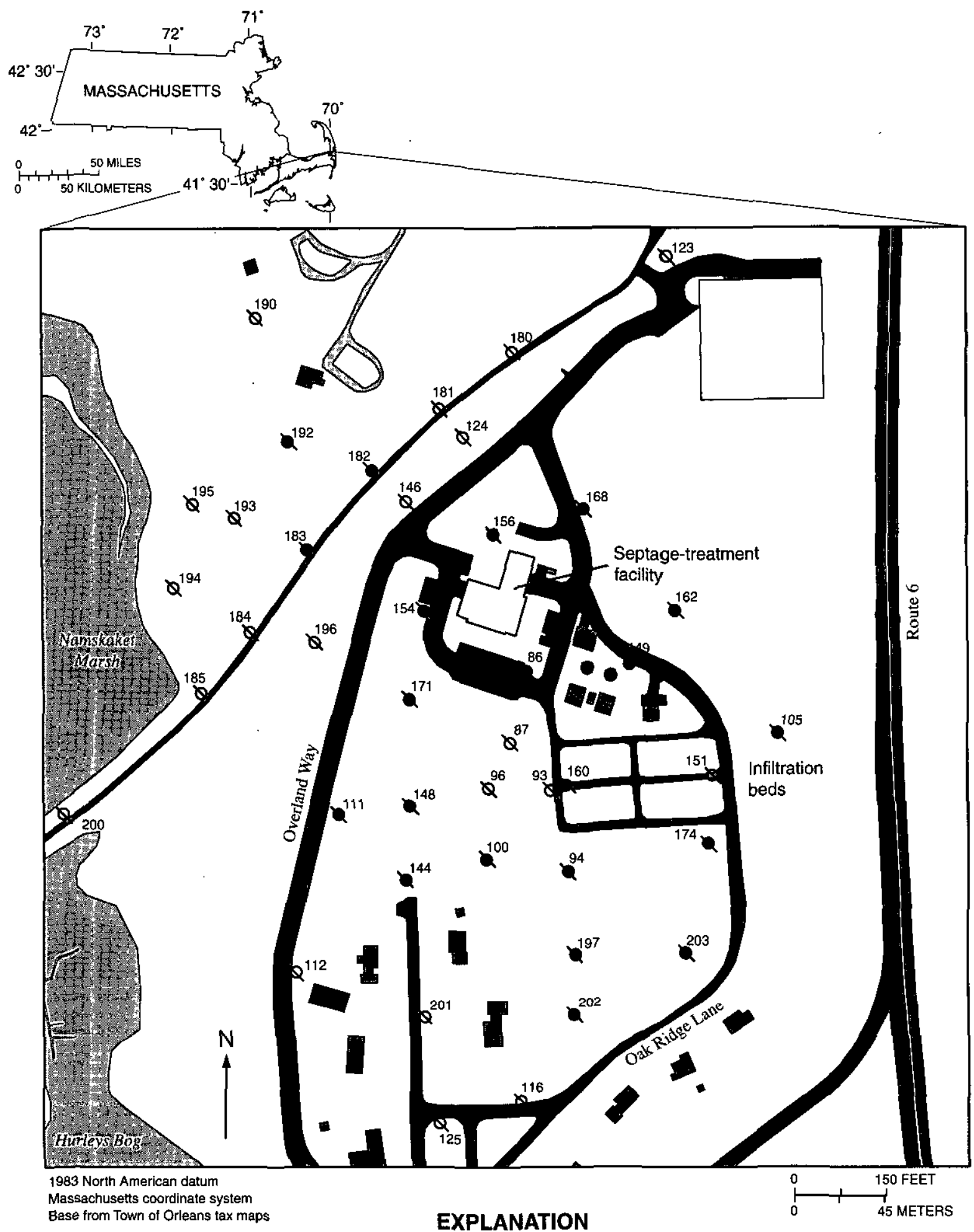

202 WELL CLUSTER SITE AND IDENTIFIER-Site of one or more observation wells completed at different depths, including wells sampled in this study (see table 1)

$Q^{125}$ WELL CLUSTER SITE AND IDENTIFIER-Site of one or more observation wells completed at different depths, wells not sampled in this study

Figure 1. Location of well-cluster sites used for water-quality sampling, Tri-Town Septage Treatment Facility, Orleans, Massachusetts. 
Table 1. Location coordinates and land-surface and screen altitudes of sampled wells, T'i-Town Septage Treatment Facility, Orleans, Massachusetts

[Latitude and longitude are given in degrees, minutes, seconds]

\begin{tabular}{|c|c|c|c|c|c|c|}
\hline $\begin{array}{l}\text { Well } \\
\text { cluster } \\
\text { site }\end{array}$ & Well Identifler & $\begin{array}{c}\text { Latitude } \\
0, " 1\end{array}$ & $\begin{array}{c}\text { Longltude } \\
0,11\end{array}$ & $\begin{array}{l}\text { Altitude of land } \\
\text { surface (feet) }\end{array}$ & $\begin{array}{l}\text { Altitude, } \\
\text { top of } \\
\text { screen } \\
\text { (feet) }\end{array}$ & $\begin{array}{l}\text { Altitude, } \\
\text { bottom of screen } \\
\text { (feet) }\end{array}$ \\
\hline 86 & OSW 84 & 414656 & 700023 & 59.8 & -16.0 & -17.0 \\
\hline \multirow[t]{2}{*}{94} & OSW 166 & 414653 & 700023 & 55.6 & -47.4 & -52.4 \\
\hline & OSW 94 & 414653 & 700023 & 55.3 & -73.6 & -74.6 \\
\hline 100 & OSW 102 & 414653 & 700024 & 41.3 & -33.2 & -34.2 \\
\hline 105 & OSW 105 & 414655 & 700018 & 65.5 & -46.5 & -51.5 \\
\hline \multirow[t]{2}{*}{111} & OSW 109 & 414654 & 700027 & 22.4 & -32.5 & -37.5 \\
\hline & OSW 111 & 414654 & 700027 & 22.4 & -69.6 & -74.6 \\
\hline 144 & OSW $144-03$ & 414653 & 700026 & 39.2 & -47.4 & -52.4 \\
\hline 148 & OSW $148-02$ & 414654 & 700026 & 38.7 & -39.8 & -44.8 \\
\hline \multirow[t]{2}{*}{149} & OSW 107 & 414656 & 700021 & 59.7 & 10.5 & 5.5 \\
\hline & OSW 149 & 414656 & 700021 & 59.8 & -12.2 & -17.2 \\
\hline 154 & OSW 155 & 414656 & 700025 & 56.4 & -46.1 & -51.1 \\
\hline 156 & OSW 161 & 414658 & 700024 & 55.1 & -23.6 & -28.6 \\
\hline 160 & OSW 160 & 414654 & 700023 & 57.2 & 12.7 & 7.7 \\
\hline \multirow[t]{2}{*}{162} & OSW 164 & 414657 & 700020 & 73.9 & 0.4 & -4.6 \\
\hline & OSW 162 & 414657 & 700020 & 73.9 & -45.1 & -50.1 \\
\hline 168 & OSW 168 & 414658 & 700022 & 50.9 & -46.1 & -51.1 \\
\hline 171 & OSW 171-02 & 414655 & 700026 & 47.0 & -16.2 & -21.2 \\
\hline \multirow[t]{2}{*}{174} & OSW 175 & 414653 & 700019 & 55.9 & -18.7 & -23.7 \\
\hline & OSW 174 & 414653 & 700019 & 55.9 & -32.0 & -37.0 \\
\hline 182 & OSW $182-02$ & 414659 & 700027 & 27.4 & -22.6 & -27.6 \\
\hline 183 & OSW 183 & 414658 & 700028 & 25.2 & -56.2 & -61.2 \\
\hline 192 & OSW 192-02 & 414700 & 700028 & 44.5 & -40.5 & -45.5 \\
\hline \multirow[t]{2}{*}{197} & OSW $197-02$ & 414651 & 700023 & 49.6 & -20.5 & -25.5 \\
\hline & OSW 197-01 & 414651 & 700023 & 49.6 & -70.5 & -75.5 \\
\hline 202 & OSW 202-02 & 414650 & 700023 & 57.0 & -39.4 & -44.0 \\
\hline 203 & OSW 203-02 & 414651 & 700020 & 55.5 & -25.9 & -30.9 \\
\hline
\end{tabular}


Table 2. Physical properties and concentrations of major inorganic consituents, nutrients, and dissolved organic carbon in ground water near the Tri-Town Septage Treatment Facility, Orleans, Massachusetts

$\left[\mathrm{CaCO}_{3}\right.$, calcium carbonate; -rep, replicate sample; -tm, sample for trace metals and volatile organic compounds; ${ }^{\circ} \mathrm{C}$. degrees Celsius; mg/L, milligrams per liter; $\mu \mathrm{g} / \mathrm{L}$, micrograms per liter; $\mu \mathrm{S} / \mathrm{cm}$, microsiemen per centimeter; $<$, concentration is less than value shown; --, no data]

\begin{tabular}{|c|c|c|c|c|c|c|c|c|}
\hline $\begin{array}{l}\text { Well } \\
\text { cluster } \\
\text { site }\end{array}$ & Well identifler & Date & Time & $\begin{array}{l}\text { Field specific } \\
\text { conductance } \\
\text { ( } \mu \mathrm{S} / \mathrm{cm} \text { at } 25^{\circ} \mathrm{C} \text { ) }\end{array}$ & $\begin{array}{l}\text { Field pH } \\
\text { (standard } \\
\text { units) }\end{array}$ & $\begin{array}{c}\text { Temperature } \\
\text { (C) }\end{array}$ & $\begin{array}{l}\text { Oxygen, } \\
\text { dissolved } \\
\text { (mg/L) }\end{array}$ & $\begin{array}{l}\text { Calcium, } \\
\text { dissoived } \\
\text { (mg/t) }\end{array}$ \\
\hline 86 & OSW 84 & $12-17-97$ & 1350 & 1,550 & 5.3 & 12.7 & 0.2 & 95 \\
\hline \multirow[t]{3}{*}{94} & OSW 166 & $12-16-97$ & 1315 & 1,710 & 5.1 & 12.6 & -- & 78 \\
\hline & OSW 166-tm & $3-10-98$ & 1400 & 1,680 & 5.0 & 13.3 & .2 & 95 \\
\hline & OSW 94 & $12-16-97$ & 1130 & 3,030 & 4.9 & 11.6 & .2 & 280 \\
\hline 100 & OSW 102 & $12-18-97$ & 1025 & 1,640 & 5.2 & 10.8 & .1 & 130 \\
\hline 105 & OSW 105 & $11-27-97$ & 1135 & 1,360 & 5.2 & 8.9 & 5.4 & 81 \\
\hline \multirow[t]{2}{*}{111} & OSW 109 & $12-17-97$ & 1130 & 298 & 5.6 & 10.6 & .0 & 13 \\
\hline & OSW 111 & $11-12-97$ & 1625 & 142 & 6.5 & 10.3 & .0 & 5.0 \\
\hline 144 & OSW 144-03 & $1-12-98$ & 1415 & 1,740 & 5.2 & 9.8 & 1.0 & 110 \\
\hline 148 & OSW 148-02 & $12-18-97$ & 1333 & 2,710 & 5.1 & 10.3 & .3 & 230 \\
\hline \multirow[t]{2}{*}{149} & OSW 107 & $11-24-97$ & 1710 & 1,180 & 5.1 & 12.7 & .4 & 55 \\
\hline & OSW 149 & $11-24-97$ & 1610 & 1,340 & 5.3 & 12.9 & .2 & 100 \\
\hline 154 & OSW 155 & $11-24-97$ & 1425 & 2,030 & 5.2 & 10.6 & .1 & 150 \\
\hline 156 & OSW 161 & $12-17-97$ & 1245 & 1,450 & 5.1 & 11.9 & .1 & 97 \\
\hline 160 & OSW 160 & $12-16-97$ & 1400 & 1,650 & 5.4 & 12.0 & - & 85 \\
\hline \multirow[t]{2}{*}{162} & OSW 162 & $11-25-97$ & 1400 & 791 & 5.9 & 10.4 & .1 & 51 \\
\hline & OSW 164 & $11-25-97$ & 1455 & 480 & 5.4 & 10.4 & 7.6 & 29 \\
\hline \multirow[t]{2}{*}{168} & OSW 168 & $11-24-97$ & 1230 & 138 & 5.2 & 10.5 & .6 & 4.7 \\
\hline & OSW 168-rep & $11-24-97$ & 1231 & 138 & 5.2 & -- & -. & 4.7 \\
\hline 171 & OSW 171-02 & $12-18-97$ & 1150 & 1,540 & 5.2 & 10.3 & .1 & 96 \\
\hline \multirow[t]{2}{*}{174} & OSW 175 & $11-25-97$ & 0955 & 1,480 & 5.4 & 11.7 & .2 & 53 \\
\hline & OSW 174 & $11-25-97$ & 0905 & 1,820 & 5.2 & 11.6 & .6 & 120 \\
\hline 182 & OSW 182-02 & $1-12-98$ & 1055 & 814 & 5.4 & 10.5 & 2.1 & 44 \\
\hline 183 & OSW 183-tm & $3-11-98$ & 1005 & 1,920 & 5.4 & 10.3 & .0 & 140 \\
\hline 192 & OSW 192-02 & $1-12-98$ & 1215 & 1,180 & 5.4 & 9.9 & 1.9 & 64 \\
\hline \multirow[t]{2}{*}{197} & OSW 197-02 & $1-06-98$ & 1255 & 1,420 & 5.2 & 10.7 & .2 & 58 \\
\hline & OSW 197-01 & $1-06-98$ & 1200 & 1,190 & 5.5 & 10.5 & 1.9 & 64 \\
\hline \multirow[t]{2}{*}{202} & OSW 202-02 & $1-06-98$ & 1410 & 1,600 & 5.3 & 10.7 & .1 & 84 \\
\hline & OSW $202-02-\mathrm{tm}$ & $3-11-98$ & 1400 & 1,600 & 5.3 & 10.6 & .05 & 89 \\
\hline 203 & OSW 203-02 & $1-06-98$ & 1025 & 2,040 & 5.4 & 11.1 & .1 & 210 \\
\hline \multicolumn{2}{|c|}{ Equipment blank I } & $11-12-97$ & 1400 & -- & - & - & -- & .1 \\
\hline \multicolumn{2}{|c|}{ Equipment blank 2} & $1-12-98$ & 1415 & 1.4 & 6.0 & -. & -- & .0 \\
\hline \multicolumn{2}{|c|}{ Rinse-water blank } & $11-12-97$ & 1345 & - & -- & -- & - & $<.02$ \\
\hline
\end{tabular}


Table 2. Physical properties and concentrations of major inorganic consituents, nutrients, and dissolved organic carbon in ground water near the Tri-Town Septage Treatment Facility, Orleans, Massachusetts-Continued

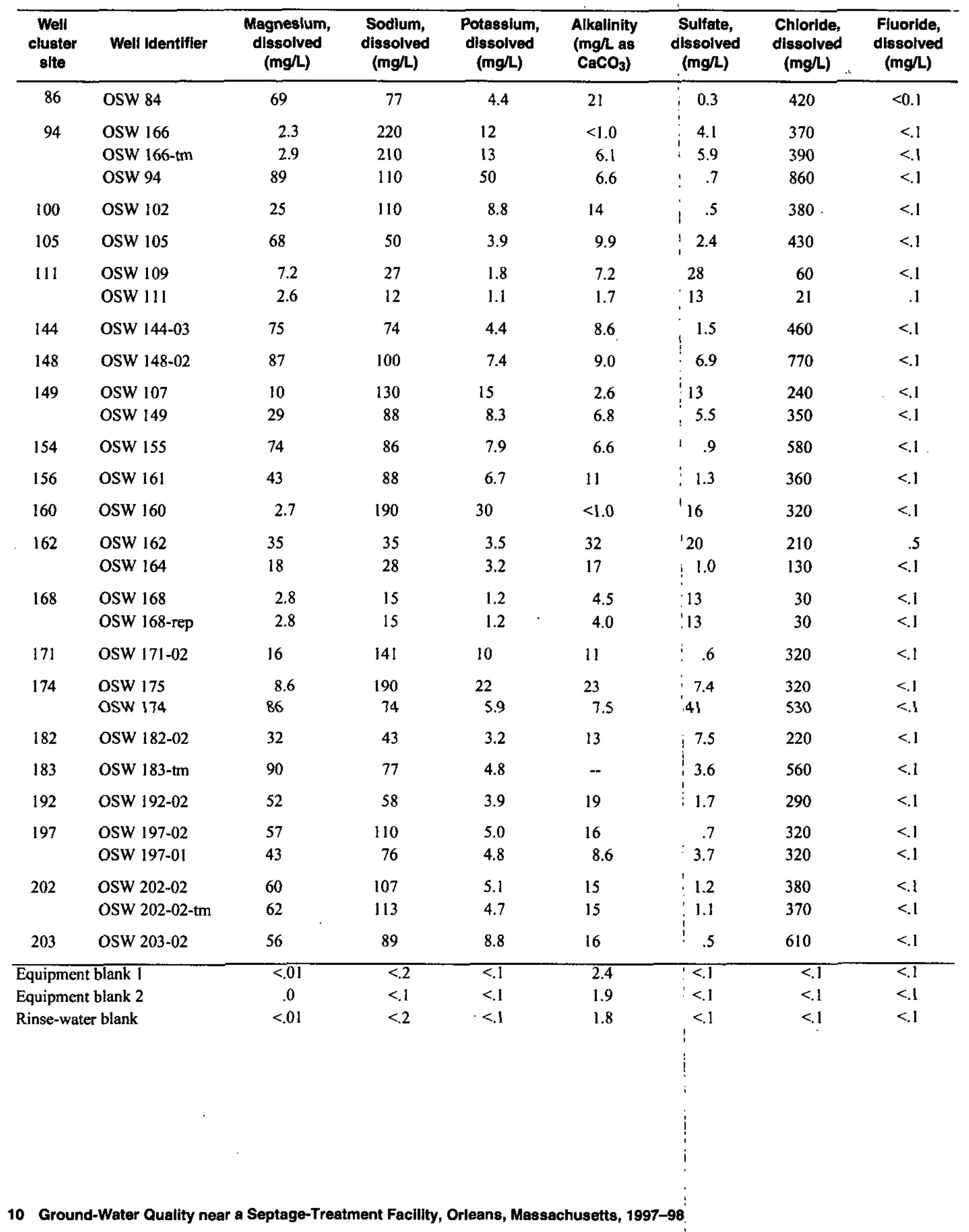


Table 2. Physical properties and concentrations of major inorganic consituents, nutrients, and dissolved organic carbon in ground wate mear the Tri-Town Septage Treatment Facility, Orleans, Massachusetts-Continued

\begin{tabular}{|c|c|c|c|c|c|c|c|c|}
\hline $\begin{array}{l}\text { Well } \\
\text { cluster } \\
\text { site }\end{array}$ & Well Identifter & $\begin{array}{l}\text { Bromide, } \\
\text { dissolved } \\
\text { (mg/L) }\end{array}$ & $\begin{array}{l}\text { Silica, } \\
\text { dissolved } \\
(\mathrm{mg} / \mathrm{l} \text { as } \\
\left.\mathrm{SiO}_{2}\right)\end{array}$ & $\begin{array}{c}\text { Nitrate, } \\
\text { dissolved } \\
\text { (calculated; } \\
\mathrm{mg} / \mathrm{L} \text { as N) }\end{array}$ & $\begin{array}{c}\text { Nitrite, } \\
\text { dissolved } \\
\text { (mg/L as N) }\end{array}$ & $\begin{array}{l}\text { Nitrite plus } \\
\text { nitrate, } \\
\text { dissolved } \\
\text { (mg/L as } N \text { ) }\end{array}$ & $\begin{array}{l}\text { Ammonia, } \\
\text { dissolved } \\
\text { (mg/L as } N \text { ) }\end{array}$ & $\begin{array}{l}\text { Ammonla plus } \\
\text { organic nitrogen, } \\
\text { dissolved } \\
\text { (mg/L as N) }\end{array}$ \\
\hline 86 & OSW 84 & 0.2 & 17 & 3.8 & 0.03 & 3.8 & $<0.02$ & 0.56 \\
\hline \multirow[t]{3}{*}{94} & OSW 166 & .3 & 11 & 63 & .23 & 63 & 3.1 & 3.8 \\
\hline & OSW 166-tm & .3 & 12 & 57 & .63 & 58 & 3.7 & 3.7 \\
\hline & OSW 94 & .3 & 12 & 46 & $<.01$ & 46 & .03 & .43 \\
\hline 100 & OSW 102 & .2 & 13 & 50 & .28 & 50 & 1.0 & 1.45 \\
\hline 105 & OSW 105 & .2 & 11 & 4.9 & $<.01$ & 4.9 & $<.02$ & $<.1$ \\
\hline \multirow{2}{*}{111} & OSW 109 & .1 & 18 & .1 & $<.01$ & .1 & $<.02$ & $<.1$ \\
\hline & osw 111 & .1 & 23 & $<.05$ & $<.01$ & $<.05$ & $<.02$ & $<.1$ \\
\hline 144 & OSW 144-03 & .3 & 11 & 31 & $<.01$ & 31 & $<.02$ & .25 \\
\hline 148 & OSW 148-02 & .3 & 13 & 33 & .03 & 33 & .20 & .83 \\
\hline \multirow[t]{2}{*}{149} & OSW 107 & .2 & 12 & 4.9 & $<.01$ & 4.9 & .95 & 1.3 \\
\hline & OSW 149 & .2 & 15 & 3.3 & .08 & 3.4 & .98 & 1.3 \\
\hline 154 & OSW 155 & .2 & 13 & 4.4 & .08 & 4.5 & .99 & 1.6 \\
\hline 156 & OSW 16! & .2 & 15 & 37 & .07 & 37 & .51 & 1.1 \\
\hline 160 & OSW 160 & .2 & 15 & 74 & .01 & 74 & 4.2 & 4.3 \\
\hline \multirow[t]{2}{*}{162} & OSW 162 & .1 & 27 & 2.4 & $<.01$ & 2.4 & $<.02$ & .13 \\
\hline & OSW 164 & .1 & 15 & 4.1 & $<.01$ & 4.1 & $<.02$ & .19 \\
\hline \multirow[t]{2}{*}{168} & OSW 168 & .1 & 16 & .1 & $<.01$ & .1 & $<.02$ & $<.1$ \\
\hline & OSW 168-rep & .1 & 16 & $<.05$ & $<.01$ & $<.05$ & $<.02$ & $<.1$ \\
\hline 171 & OSW 171-02 & .3 & 13 & 56 & .42 & 56 & 3.3 & 3.8 \\
\hline \multirow[t]{2}{*}{174} & OSW 175 & .2 & 11 & 44 & .37 & 44 & 8.0 & $<.1$ \\
\hline & OSW 174 & .3 & 18 & 8.1 & $<.01$ & 8.1 & $<.02$ & .52 \\
\hline 182 & OSW $182-02$ & .1 & 18 & 8.1 & $<.01$ & 8.1 & $<.02$ & .17 \\
\hline 183 & OSW 183-tm & -- & 16 & 28 & .01 & 28 & $<.02$ & .57 \\
\hline 192 & OSW 192-02 & .2 & 15 & 22 & $<.01$ & 22 & $<.02$ & .20 \\
\hline \multirow[t]{2}{*}{197} & OSW 197-02 & .2 & 15 & 45 & .37 & 45 & $<.02$ & .24 \\
\hline & OSW 197-01 & .2 & 16 & 14 & $<.01$ & 14 & $<.02$ & .14 \\
\hline \multirow{2}{*}{202} & OSW 202-02 & .2 & 15 & 44 & .09 & 44 & $<.02$ & .56 \\
\hline & OSW 202-02-tm & .3 & 15 & 47 & .10 & 47 & $<.02$ & .66 \\
\hline 203 & OSW 203-02 & .3 & 18 & 37 & $<.01$ & 37 & .58 & 1.4 \\
\hline \multicolumn{2}{|c|}{ Equipment blank I } & $<.01$ & 1.4 & $<.05$ & $<.01$ & $<.05$ & $<.02$ & $<.1$ \\
\hline \multicolumn{2}{|c|}{ Equipment blank 2} & $<.01$ & $<.1$ & $<.05$ & $<.01$ & $<.05$ & $<.02$ & $<.1$ \\
\hline \multicolumn{2}{|c|}{ Rinse-water blank } & $<.01$ & 1.4 & $<.05$ & $<.01$ & $<.05$ & $<.02$ & $<.1$ \\
\hline
\end{tabular}


Table 2. Physical properties and concentrations of major inorganic consituents, nutrients, and dissolved organic carbon in ground water near the Tri-Town Septage-Treatment Facility, Orleans, Massachusetts-Continued

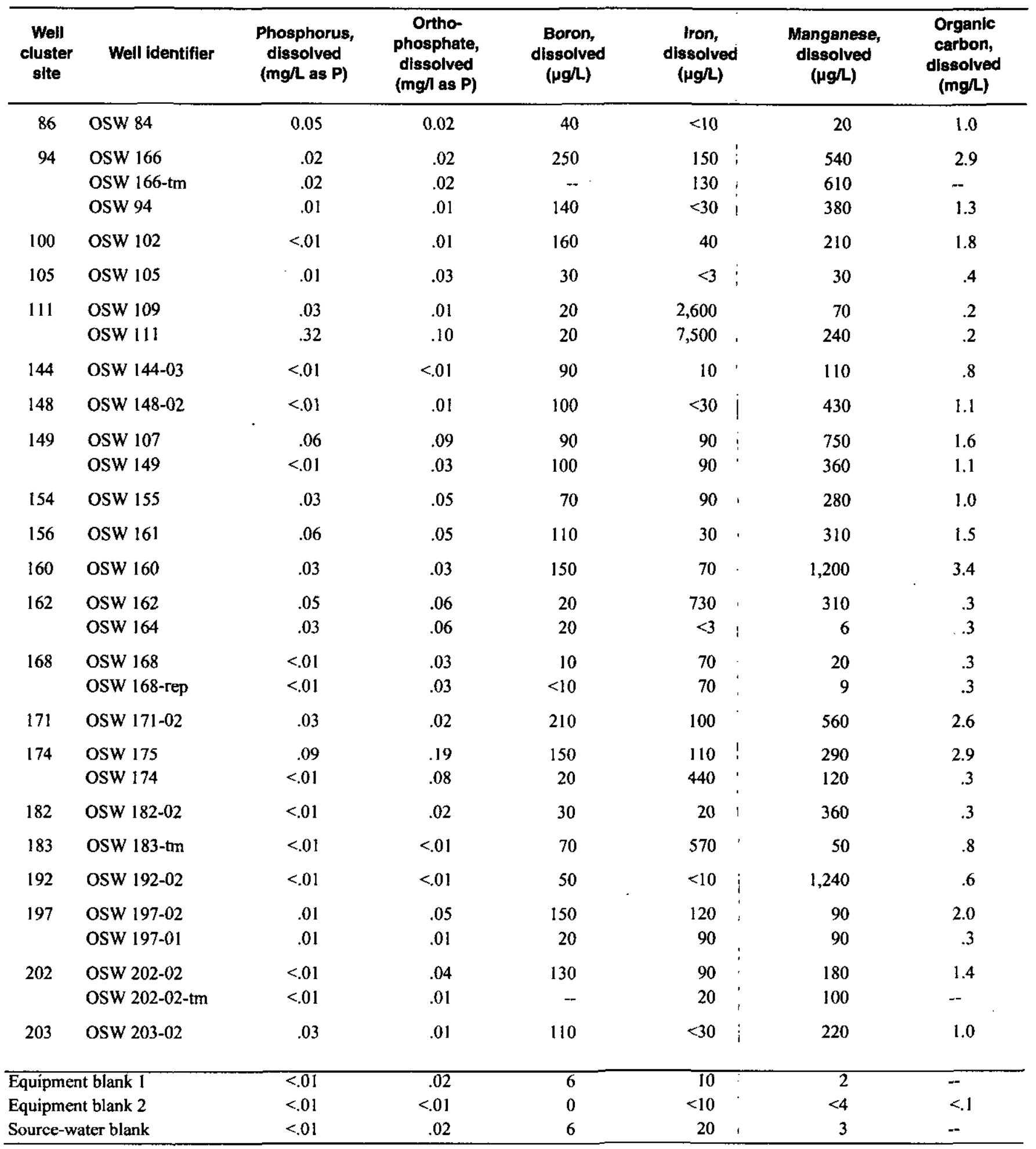


Table 3. Concentrations of trace metals in ground water near the Tri-Town Septage Treatment Facility, Orleans, Massachusetts

[Ground-water well OSW 183, 3-10-98: Sample was split in the field and splits were analyzed separately. Concentrations in micrograms per liter; $<$, concentration is less than value shown]

\begin{tabular}{|c|c|c|c|c|c|}
\hline \multirow{3}{*}{ Trace metal } & \multicolumn{4}{|c|}{ Ground-water wells } & \multirow{3}{*}{$\begin{array}{c}\text { Quality-assurance sample } \\
\begin{array}{c}\text { Equipment blank } \\
\text { 3-11-98 }\end{array}\end{array}$} \\
\hline & \multirow{2}{*}{\multicolumn{2}{|c|}{$\begin{array}{c}\text { Site } 183 \\
\text { OSW } 183 \\
3-10-98\end{array}$}} & \multirow{3}{*}{$\begin{array}{c}\text { Site } 94 \\
\begin{array}{c}\text { OSW } 166 \\
3-10-98\end{array} \\
26\end{array}$} & \multirow{3}{*}{$\begin{array}{c}\text { Site } 202 \\
\begin{array}{c}\text { OSW 202-02 } \\
3-11-98\end{array} \\
33\end{array}$} & \\
\hline & & & & & \\
\hline 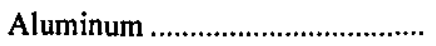 & 9.9 & 9.4 & & & $<1.0$ \\
\hline Antiminy & $<1.0$ & $<1.0$ & $<1.0$ & $<1.0$ & $<1.0$ \\
\hline 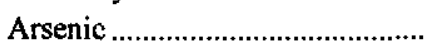 & $<1.0$ & $<1.0$ & $<1.0$ & $<1.0$ & $<1.0$ \\
\hline 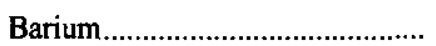 & 58 & 58 & 34 & 41 & $<1.0$ \\
\hline 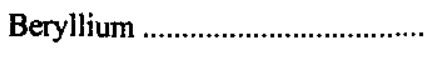 & $<1.0$ & $<1.0$ & $<1.0$ & $<1.0$ & $<1.0$ \\
\hline 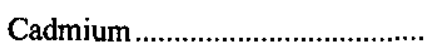 & $<1.0$ & 1.0 & $<1.0$ & $<1.0$ & $<1.0$ \\
\hline 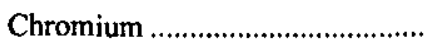 & 1.3 & 1.4 & 2.4 & 2.3 & $<1.0$ \\
\hline 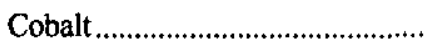 & 1.5 & 1.6 & 8.2 & 1.9 & $<1.0$ \\
\hline 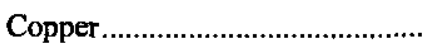 & $<1.0$ & $<1.0$ & 1.6 & 1.0 & $<1.0$ \\
\hline 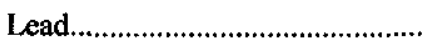 & $<1.0$ & $<1.0$ & $<1.0$ & $<1.0$ & $<1.0$ \\
\hline 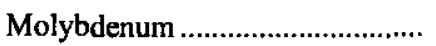 & $<1.0$ & $<1.0$ & $<1.0$ & $<1.0$ & $<1.0$ \\
\hline 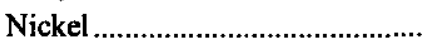 & 31 & 31 & 4.3 & 3.8 & $<1.0$ \\
\hline 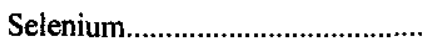 & $<1.0$ & $<1.0$ & $<1.0$ & $<1.0$ & $<1.0$ \\
\hline Silver & $<1.0$ & $<1.0$ & $<1.0$ & $<1.0$ & $<1.0$ \\
\hline 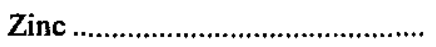 & 31 & 31 & 5.5 & 3.8 & $<1.0$ \\
\hline 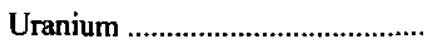 & $<1.0$ & $<1.0$ & $<1.0$ & $<1.0$ & $<1.0$ \\
\hline
\end{tabular}


Table 4. Concentrations of volatile organic compounds in ground water near the Tri-Town SeptageTreatment Facility, Orleans, Massachusetts

[Concentrations in micrograms per liter; <, concentration is less than value shown

\begin{tabular}{|c|c|c|c|c|c|c|}
\hline \multirow{3}{*}{ Volatile organic compound } & \multicolumn{3}{|c|}{ Ground-water wells } & \multicolumn{3}{|c|}{ Quality-assurance samples } \\
\hline & \multirow{2}{*}{ 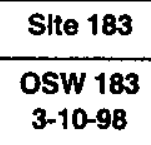 } & \multirow{2}{*}{$\begin{array}{c}\text { Site } 94 \\
\text { OSW } 166 \\
3-11-98\end{array}$} & \multirow{2}{*}{$\begin{array}{c}\text { Site } 202 \\
\text { OSW 202-02 } \\
\text { 3-11-98 }\end{array}$} & \multirow{2}{*}{\multicolumn{2}{|c|}{$\begin{array}{l}\text { Equipment blank } \\
3-11-98\end{array}$}} & \multirow[b]{2}{*}{ Laboratory blank } \\
\hline & & & & & & \\
\hline Acetone & $<5$ & $<5$ & $<5$ & 1 & 7 & $<5$ \\
\hline Acrolein & $<250$ & $<250$ & $<250$ & & $<250$ & $<250$ \\
\hline Acrylonitrile & $<2$ & $<2$ & $<2$ & & $<2$ & $<2$ \\
\hline Benzene & $<.2$ & $<.2$ & $<.2$ & & $<.2$ & $<.2$ \\
\hline 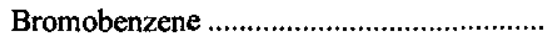 & $<.2$ & $<.2$ & $<.2$ & $:$ & $<.2$ & $<.2$ \\
\hline Bromochloromethane & $<.2$ & $<.2$ & $<.2$ & & $<.2$ & $<.2$ \\
\hline Bromodichloromethane & $<.2$ & $<.2$ & $<.2$ & $\cdot$ & $<.2$ & $<.2$ \\
\hline Bromoethene & $<.2$ & $<.2$ & $<.2$ & 1 & $<.2$ & $<.2$ \\
\hline Bromoform & $<.2$ & $<, 2$ & $<.2$ & '. & $<.2$ & $<.2$ \\
\hline Bromomethane & $<.2$ & $<.2$ & $<.2$ & & $<.2$ & $<.2$ \\
\hline 2-Butanone & $<5$ & $<5$ & $<5$ & & $<5$ & $<5$ \\
\hline$n$-Butylbenzene & $<.2$ & $<.2$ & $<.2$ & & $<.2$ & $<.2$ \\
\hline sec-Butylbenzene & $<.2$ & $<.2$ & $<.2$ & & $<.2$ & $<.2$ \\
\hline tert-Butylbenzene & $<.2$ & $<.2$ & $<.2$ & & $<.2$ & $<.2$ \\
\hline Carbon disulfide & $<.2$ & $<.2$ & $<.2$ & ' & $<.2$ & $<.2$ \\
\hline 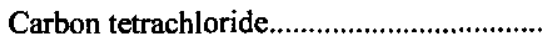 & $<.2$ & $<.2$ & $<.2$ & . & $<.2$ & $<.2$ \\
\hline Chlorobenzene & $<.2$ & $<.2$ & $<.2$ & & $<2$ & $<.2$ \\
\hline 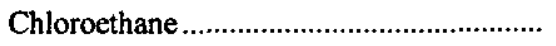 & .4 & .2 & .4 & . & .5 & $<.2$ \\
\hline 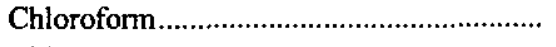 & $<.2$ & $<.2$ & .2 & . & $<.2$ & $<.2$ \\
\hline Chloromethane & 3.2 & 1.9 & 2.9 & & 2.9 & $<.2$ \\
\hline 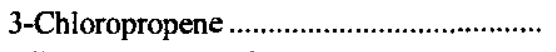 & $<.5$ & $<.5$ & $<.5$ & ' & $<.5$ & $<.5$ \\
\hline Dibromochloromethane ................................ & $<.2$ & $<.2$ & $<.2$ & & $<.2$ & $<.2$ \\
\hline Dibromochloropropane (DBCP) ...................... & $<1$ & $<1$ & $<1$ & ' & $<1$ & $<1$ \\
\hline 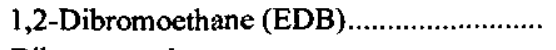 & $<.2$ & $<.2$ & $<.2$ & & $<.2$ & $<.2$ \\
\hline Dibromomethane & $<.2$ & $<.2$ & $<.2$ & & $<.2$ & $<.2$ \\
\hline 1,2-Dichlorobenzene & $<.2$ & $<.2$ & $<.2$ & $\vdots$ & $<.2$ & $<.2$ \\
\hline 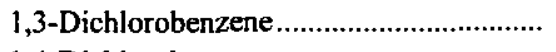 & $<.2$ & $<.2$ & $<.2$ & : & $<.2$ & $<.2$ \\
\hline 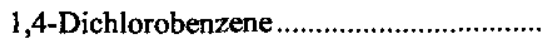 & $<.2$ & $<.2$ & $<.2$ & 1 & $<.2$ & $<.2$ \\
\hline 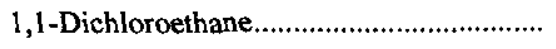 & $<.2$ & $<.2$ & $<.2$ & i & $<2$ & $<.2$ \\
\hline 1,2 Dichloroethane & $<.2$ & $<.2$ & $<.2$ & ! & $<.2$ & $<.2$ \\
\hline 1,1-Dichloroethene & $<.2$ & $<.2$ & $<.2$ & 1 & $<.2$ & $<.2$ \\
\hline 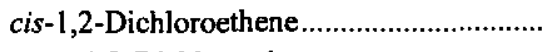 & $<.2$ & $<.2$ & $<.2$ & & $<.2$ & $<.2$ \\
\hline 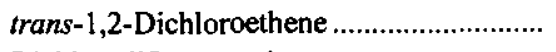 & $<.2$ & $<.2$ & $<.2$ & $\vdots$ & $<.2$ & $<.2$ \\
\hline 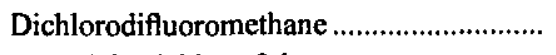 & ${ }^{1} .23$ & ${ }^{1} .77$ & 1.42 & 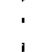 & $<.2$ & $<.2$ \\
\hline 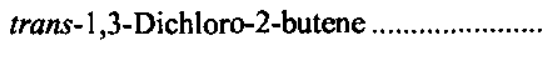 & $<5$ & $<5$ & $<5$ & 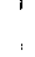 & $<5$ & $<5$ \\
\hline 1,2-Dichloropropane & $<.2$ & $<.2$ & $<.2$ & & $<.2$ & $<.2$ \\
\hline 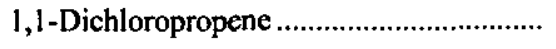 & $<.2$ & $<.2$ & $<.2$ & & $<.2$ & $<.2$ \\
\hline 1,3-Dichloropropene & $<.2-$ & $<.2$ & $<.2$ & ' & $<.2$ & $<.2$ \\
\hline 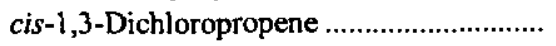 & $<.2$ & $<.2$ & $<.2$ & & $<.2$ & $<.2$ \\
\hline 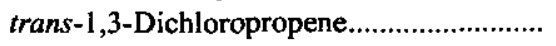 & $<.2$ & $<.2$ & $<.2$ & & $<.2$ & $<.2$ \\
\hline
\end{tabular}


Table 4. Concentrations of volatile organic compounds in ground water near the Tri-Town SeptageTreatment Facility, Orleans, Massachusetts-Continued

\begin{tabular}{|c|c|c|c|c|c|}
\hline \multirow{3}{*}{ Volatile organic compound } & \multicolumn{3}{|c|}{ Ground-water wells } & \multicolumn{2}{|c|}{ Quality-assurance samples } \\
\hline & \multirow{2}{*}{$\begin{array}{c}\text { Site } 183 \\
\text { OSW } 183 \\
3-10-98\end{array}$} & \multirow{2}{*}{$\begin{array}{c}\text { Site } 94 \\
\text { OSW } 166 \\
3-11-98\end{array}$} & \multirow{2}{*}{$\begin{array}{c}\text { Site } 202 \\
\text { OSW 202-02 } \\
3-11-98\end{array}$} & \multirow{2}{*}{$\begin{array}{l}\text { Equipment blank } \\
\text { 3-11-98 }\end{array}$} & \multirow[b]{2}{*}{ Laboratory blank } \\
\hline & & & & & \\
\hline 2,2-Dichloropropene & $<.2$ & $<.2$ & $<.2$ & $<.2$ & $<.2$ \\
\hline 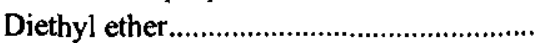 & $<.2$ & $<.2$ & $<.2$ & $<.2$ & $<.2$ \\
\hline 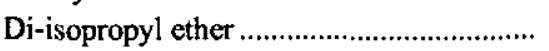 & $<.2$ & $<.2$ & $<.2$ & $<.2$ & $<.2$ \\
\hline Ethylbenzene & $<.2$ & $<.2$ & $<.2$ & $<.2$ & $<.2$ \\
\hline 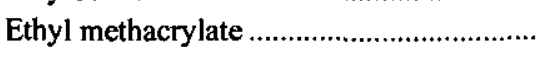 & $<1$ & $<1$ & $<1$ & $<1$ & $<1$ \\
\hline Ethyl tert-butyl ether & $<.2$ & $<.2$ & $<.2$ & $<.2$ & $<.2$ \\
\hline Ethyl tert-pentyl methyl ............................... & $<.2$ & $<.2$ & $<.2$ & $<.2$ & $<.2$ \\
\hline Hexachlorobutadiene......................................... & $<.2$ & $<.2$ & $<.2$ & $<.2$ & $<.2$ \\
\hline 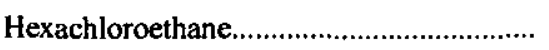 & $<.2$ & $<.2$ & $<.2$ & $<.2$ & $<.2$ \\
\hline 2-Hexanone & $<5$ & $<5$ & $<5$ & $<5$ & $<5$ \\
\hline 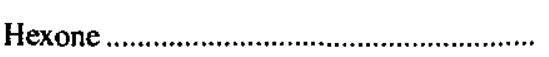 & $<5$ & $<5$ & $<5$ & $<5$ & $<5$ \\
\hline 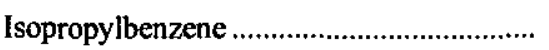 & $<.2$ & $<.2$ & $<.2$ & $<.2$ & $<.2$ \\
\hline$p$-Isopropyltoluene & $<.2$ & $<.2$ & $<.2$ & $<.2$ & $<.2$ \\
\hline 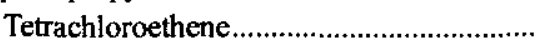 & $<.2$ & $<.2$ & $<.2$ & $<.2$ & $<.2$ \\
\hline Methyl acrylate & $<2$ & $<2$ & $<2$ & $<2$ & $<2$ \\
\hline Methyl acrylonitrile & $<2$ & $<2$ & $<2$ & $<2$ & $<2$ \\
\hline Methyl iodide & $<.2$ & $<.2$ & $<.2$ & $<.2$ & $<.2$ \\
\hline Methyl methacrylate & $<1$ & $<1$ & $<1$ & $<1$ & $<1$ \\
\hline Methyl tert-butyl ether (MTBE) ................... & $<.2$ & $<.2$ & $<.2$ & $<.2$ & $<.2$ \\
\hline Methylene chloride & $<.2$ & $<.2$ & $<.2$ & $<.2$ & $<.2$ \\
\hline Naphthalene & $<2$ & $<.2$ & $<.2$ & $<.2$ & $<.2$ \\
\hline$n$-Propylbenzene & $<.2$ & $<.2$ & $<.2$ & $<.2$ & $<.2$ \\
\hline Styrene & $<.2$ & $<.2$ & $<.2$ & $<.2$ & $<.2$ \\
\hline 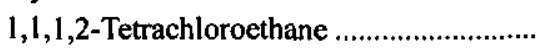 & $<.2$ & $<.2$ & $<.2$ & $<.2$ & $<.2$ \\
\hline 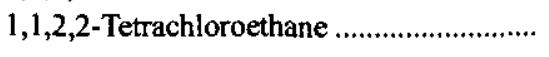 & $<.2$ & $<.2$ & $<.2$ & $<.2$ & $<.2$ \\
\hline 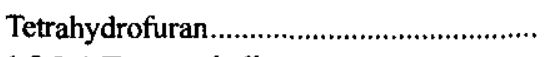 & $<5$ & $<5$ & $<5$ & $<5$ & $<5$ \\
\hline 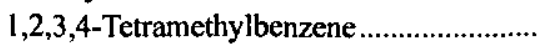 & $<.2$ & $<.2$ & $<.2$ & $<.2$ & $<.2$ \\
\hline 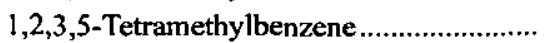 & $<.2$ & $<.2$ & $<.2$ & $<.2$ & $<.2$ \\
\hline 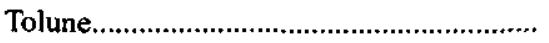 & $<.2$ & $<.2$ & $<.2$ & $<.2$ & $<.2$ \\
\hline Trichlorofluoromethane & $<.2$ & $<.2$ & $<.2$ & $<.2$ & $<.2$ \\
\hline 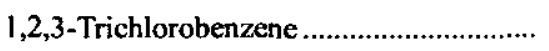 & $<.2$ & $<.2$ & $<.2$ & $<.2$ & $<.2$ \\
\hline 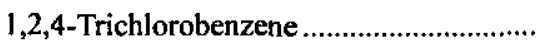 & $<.2$ & $<.2$ & $<.2$ & $<.2$ & $<.2$ \\
\hline 1,1,1-Trichloroethane & .2 & $<.2$ & $<.2$ & $<.2$ & $<.2$ \\
\hline $1,1,2$-Trichloroethane & $<.2$ & $<.2$ & $<.2$ & $<.2$ & $<.2$ \\
\hline Trichloroethene & $<.2$ & $<.2$ & $<.2$ & $<.2$ & $<.2$ \\
\hline 1,1,2-Trichlorotrifluoroethane ......................... & $<.2$ & $<.2$ & $<.2$ & $<.2$ & $<.2$ \\
\hline 1,2,3-Trichloropropane & $<.2$ & $<.2$ & $<.2$ & $<.2$ & $<.2$ \\
\hline 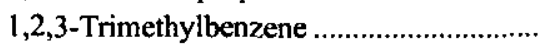 & $<.2$ & $<.2$ & $<.2$ & $<.2$ & $<.2$ \\
\hline 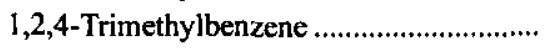 & $<.2$ & $<.2$ & $<.2$ & $<.2$ & $<.2$ \\
\hline 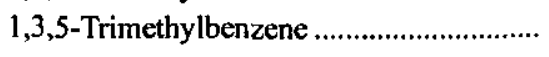 & $<.2$ & $<.2$ & $<.2$ & $<.2$ & $<.2$ \\
\hline Vinyl chloride & $<.2$ & $<.2$ & $<.2$ & $<.2$ & $<.2$ \\
\hline$m$ - and $p$-Xylene & $<.2$ & $<.2$ & $<.2$ & $<.2$ & $<.2$ \\
\hline o-Xylene & $<.2$ & $<.2$ & $<.2$ & $<.2$ & $<.2$ \\
\hline
\end{tabular}

1 Concentration is estimated. 